\title{
Über die bei der Uranspaltung noch zu erwartenden Bruchstücke*
}

\author{
Von W. Seelmann-Eggebert und F. Strassmann \\ Aus dem Kaiser-Wilhelm-Institut für Chemie, Tailfingen \\ (Z. Naturforschg. 2a, 80-86 [1947]; eingegangen am 31. Oktober 1946)
}

\begin{abstract}
Die Arbeit gibt einen Überblick über die bisher bekannt gewordenen Uranspaltprodukte. Alle einzelnen Isotope werden im Hinblick auf ihre Einordnung in das System der Atomkerne diskutiert und Gründe für das wahrscheinliche Auftreten weiterer Isotope bei der Spaltung aufgeführt. Die mit Hilfe von chemischen Methoden bisher erzielten Ergebnisse werden zusammengefaßt. Abschließend wird auf Probleme und noch nicht geklärte Fragen der Spaltung des Urans hingewiesen.
\end{abstract}

\section{T} rägt man die bei der Uranspaltung aufgefundenen aktiven Isotope in das System der Atomkerne ein, so zeigt sich bereits bei oberflächlicher Betrachtung, daß schon der überwiegende Teil der überhaupt möglichen Spaltstücke bekannt ist. Über die Art und Weise, wie die Massenzahlen der einzelnen Uranbruchstücke festgestellt worden sind, sei hier auf die Originalarbeiten verwiesen ${ }^{1}$.

Die mit physikalischen Methoden durchgeführten Untersuchungen ${ }^{2,3,4,5}$ der bei der Spaltung der schweren Atomkerne mit verlangsamten und mit $\mathrm{Ra} / \mathrm{Be}-\mathrm{Neutronen}$ auftretenden Massen der Bruchstücke zeigen, daß sämtliche Kernmassen von etwa 66 bis 170 aufzutreten scheinen. Ob sich in diesen Grenzen aktive Isobare aller Massenzahlen feststellen lassen werden, ist damit nicht gesagt. Es könnten als primäre Bruchstücke auch sofort stabile Isotope angenommen werden; allerdings ist es viel wahrscheinlicher, daß ein oder mehrere aktive Isobare bei jeder Massenzahl auftreten, weil die Uranbruchstücke sofort nach der Spaltung einen ziemlich großen Neutronenüberschuß haben müssen, da eine direkte Abspaltung von Neutronen nur in verhältnismäßig kleinem Ausmaße erfolgt. Ein solcher Überschuß wird

\footnotetext{
* Am Schluß dieses Heftes befindet sich ein Auszug aus einem amerikanischen Bericht über die Uranspaltprodukte vom November 1946. Alle von uns erwähnten Spaltprodukte sind im Kaiser-Wilhelm-Institut für Chemie geprüft bzw. aufgefunden worden. Einige wenige unserer neueren Angaben sind im amerikanischen Bericht nicht enthalten, während sämtliche Angaben des amerikanischen Berichtes über die bei der Spaltung auftretenden Kerne mit einer Ordnungszahl größer als 59 und kleiner als 35 , bzw. über solche Kerne, welchẻ nur mit sehr geringer Intensität bei der Uranspaltung auftreten, uns bisher unbekannt waren.
}

bekanntlich durch eine Reihe von $\beta$-Umwandlungen ausgeglichen. Die Annahme, daß alle Bruchstücke zuerst einen Neutronenüberschuß haben, findet eine Stütze in der Tatsache, daß bisher keine Positronenstrahler unter den Spaltprodukten aufgefunden wurden und bis heute auch kein aktives Spaltprodukt, welches eine höhere Ordnungszahl besitzt als das stabile Isobar. Veranschaulicht man sich dieses Ergebnis an Hand des Energietales der Atomkerne, so läßt sich erkennen, daß die Grenze der Spaltprodukte in der ersten Senke, also dem ersten Energieminimum der stabilen Isotope, verläuft. Unter erster Senke wird, von der Seite der $\beta$-strahlenden Isotope her gesehen, das erste stabile Isobar verstanden. Bei der Spaltung wurden bis heute aktive Isotope jenseits dieser durch das erste stabile Isotop gegebenen Grenze nicht aufgefunden. Diese Grenze ist in Tab. 1 in Form einer starken Linie hinter dem ersten stabilen Isobar einer jeden Massenzahl eingezeichnet. Innerhalb dieser Begrenzung sind natürlich unter den Spaltprodukten auch isomere Kerne sonst stabiler Isotope zu erwarten.

Bisher ist möglicherweise nur eine Ausnahme von dieser Regel bekannt. P olessitski und Mitarbeiter ${ }^{6}$ glauben, bei der Thoriumspaltung,

1 Kernphysikalische Tabellen von J. M a t t a u c h u. S. F l ü g g e, Berlin, Springer 1942; G. T. Se a b o r g, Table of Isotopes, Rev. mod. Physics 16, Nr. 1 [1944].

${ }^{2}$ W. Jentschke u. F. Prankl, Z Physik 119, 696 [1942].

${ }^{3}$ A. Flammersfeld, W. Gentner, A. Jen sen, Z. Physik 120, 450 [1943].

4 W. Jents ch ke, Z. Physik 120, 165 [1943].

5 O. B r u n a, Diss. Wien [1941].

6 A. Polessitsky u. Nemerowsky, C. R. [Doklady] Akad. Sci. URSS 28, 217 [1940]. 
für welche das gleiche gelten müßte, das auf Grund anderer Kernprozesse lange bekannte Brom von 4,4 Stdn. Halbwertszeit der Massenzahl 80 aufgefunden zu haben. Zu diesem BromIsotop existiert ein stabiles Isobar niedrigerer Ordnungszahl, das ${ }^{80}$ Selen. Bei Nachprüfung der Brom-Aktivitäten ließ sich bei der Spaltung von Thorium und auch von Uran keine Andeutung für das Vorhandensein dieses Brom-Isotops finden. Die Nachprüfung soll jedoch, wenn möglich, mit einer stärkeren Neutronenquelle wiederholt werden. Bisher konnten die Versuche nur mit den Neutronen einer $\mathrm{Ra} / \mathrm{Be}-\mathrm{Quelle}$ durchgeführt werden.

In Tab. 1 sind eine Reihe von Massenzahlen aufgeführt, zu welchen bisher noch kein Uranspaltisotop aufgefunden werden konnte: Man kann in den meisten Fällen begründen, warum sich gerade diese bisher dem Nachweis entzogen haben. Die Gründe hierfür sollen im folgenden bei den einzelnen Elementen näher erläutert werden. Vor allem fehlen noch dort aktive Isotope, wo die Intensitäten der Spaltprodukte relativ sehr klein sind, also bei Bruchstücken, deren Massenzahlen wenig, wie z. B. in der Nähe der Massenzahl 118, oder besonders stark voneinander unterschieden sind, wie Isotope unterhalb 83 und oberhalb 146, oder dort, wo die schnelle chemische Abtrennung schwierig durchzuführen ist. Auch Isotope mit extrem langen Halbwertszeiten sind wegen ihrer geringen Aktivität schwer aufzufinden. Diese Umstände sprechen alle dafür, daß es wohl noch gelingen wird, die nach den oben angedeuteten Gesetzmäßigkeiten noch fehlenden aktiven Spaltstücke aufzufinden, wenn keine bisher noch nicht erkannten kernphysikalischen Regeln ihr Entstehen verbieten. Vor allem aber sind auch unter- und oberhalb der in Tab. 1 aufgeführten Elemente noch aktive Spaltisotope zu erwarten, zu deren Nachweis allerdings wesentlich stärkere Neutronenquellen zur Verfügung stehen müssen, als sie bei unseren Arbeiten benutzt worden sind. Auf Grund der oben zitierten physikalischen Meßergebnisse sollten aktive Isotope der Elemente von Nickel bis etwa zum Holmium aufgefunden werden können. Ihre Intensität ist im Verhältnis zu den bekannten Uranspaltprodukten, wie bereits erwähnt, als äußerst gering anzunehmen.

Unter den bisher chemisch isolierten Spaltprodukten tritt als Element niedrigster Ordnungszahl das Brom auf. Es ließen sich Brom-Isotope der Masse 83, 84, 85 und 87 feststellen. Ein Isotop der Masse 86 ist noch unbekannt. Der Grund dafür ist in der vermutlich kurzen Halbwertszeit zu suchen. Mit steigendem Neutronenüberschuß, d. h. mit steigender Massenzahl der primären Brom-Isotope, nimmt die Halbwertszeit von 140 Min. bis auf etwa 50 Sek. kontinuierlich ab. Auch bei anderen Elementen ist im allgemeinen ein derartiger Zusammenhang zwischen Halbwertszeit und Neutronenüberschuß zu verzeichnen, jedoch muß betont werden, daß es von dieser Regel zahlreiche Ausnahmen gibt. Im Falle des ${ }^{86}$ Broms ist also möglicherweise mit einer Halbwertszeit zwischen 50 und 180 Sek. zu rechnen. In diesem Falle würde es schwer sein, ein derartiges Brom zu erfassen, wenn nicht sehr starke Strahlenquellen in der Nähe des Arbeitsplatzes zur Verfügung stehen. Aber auch für den Fall, daß die Halbwertszeit des ${ }^{86}$ Broms extrem lang wäre, würde sein Nachweis schwierig sein.

Beim Krypton wurde bisher die Existenz von zehn Isotopen nachgewiesen, von denen sieben als vermutlich primäre Bruchstücke anzusehen sind, da bisher keine Muttersubstanzen zu ihnen gefunden wurden. Die drei aus Brom-Isotopen nachgebildeten Krypton-Isotope liegen eindeutig fest, genau wie die beiden Krypton-Isotope mit den Halbwertszeiten von 175 und 2,5 Min. Den restlichen Krypton-Isotopen wurden die Massenzahlen 90, 91, 92, 94 und 96 zugeordnet. Die Halbwertszeiten konnten zwar nicht angegeben werden, da die betreffenden Substanzen zu kurzlebig sind; doch wurde ihre Existenz nachgewiesen und ihre Masse durch die Möglichkeit, ihre Folgeprodukte wenigstens zum Teil bestimmten Massenzahlen zuordnen zu können, festgelegt. Bei den Massenzahlen 92 und 94 ist die Zuordnung nicht sicher.

Beim Element 37, dem Rubidium, wurden acht Isotope nachgewiesen. Für drei konnten Halbwertszeit und Massenzahl angegeben werden.

Beim Strontium liegen bisher sieben Isotope vor. Drei davon konnten bestimmten Massenzahlen zugeordnet werden. Die Isotope von 2,7 Stdn., 2 Min. und 7 Min. verteilen sich auf die Massen 92, 94 und 96, während ein 6-10-Tage-Strontium noch nicht sicher ist?

Beim Yttrium sind gleichfalls sieben Isotope vorhanden. Die Yttrium-Isotope mit den Massenzahlen 90 und 91 sind eindeutig festgelegt; bei der Masse 91 ist eine Isomerie gefunden worden. Das 50-Min.Yttrium ist wegen seiner $\gamma$-Strahlung, die beim 57-Tage-Yttrium nicht nachzuweisen ist, als der angeregte Zustand anzusehen ${ }^{7}$. $\mathrm{Zu}$ den Yttrium-Isotopen von 11,6 Stdn., 3,5 Stdn. und 20 Min. Halbwertszeit scheinen die Massen 92, 94 und 96 zu gehören. Eine endgültige Einzelzuordnung dieser drei Isotope ist allerdings noch nicht möglich.

Die Stellung und die Existenz des aufgeführten etwa 100-Tage-Yttriums ist nicht sicher ${ }^{7}$. Auf jeden Fall muß entweder das 11,6-Stdn.- oder das 100-Tage-

7 Noch unveröffentlichte Arbeiten von W. S e e lmann u. F. Straßmann. 
Yttrium eine aktive Tochtersubstanz nachbilden. Die Verhältnisse der Reihen bei den Massenzahlen 97 und 98 sind also noch recht unklar.

Beim Zirkon wurden bisher nur zwei Isotope zugeordnet, und zwar den Massen 93 und 95. Diese beiden Zirkon-Isotope treten anscheinend primär auf, da eine Muttersubstanz bisher bei beiden nicht gefunden wurde. Ein weiteres Zirkon-Isotop von 26 Tagen Halbwertszeit ist noch nicht bestätigt worden; wahrscheinlich hat es eine kurzlebige Tochtersubstanz und besitzt die Masse 97 oder vielleicht 98.

$\mathrm{Zu}$ den Massenzahlen 93 und 95 gehören Niob-Isotope von 55 Tagen und 75 Min. Halbwertszeit. Ein weiteres Niob-Isotop sehr langer Halbwertszeit und weicher Strahlung wurde nachgewiesen ${ }^{7}$. Es muß - eine der Massenzahlen 97, 98 bzw. 100 besitzen. Weitere vermutlich auftretende aktive Niob-Isotope sind noch unbekannt, vielleicht sind sie kurzlebig. Da noch keine Niob-Abtrennung aus dem Uran kurz nach dem Ende der Bestrahlung durchgeführt worden ist, kann hierüber nichts ausgesagt werden.

Drei aktive Isotope des Molybdäns wurden den Massenzahlen 99, 101 und 105 zugeordnet. Ein weiteres Isotop von $12 \mathrm{Min}$. Halbwertszeit liegt in bezug auf seine Massenzahl noch nicht fest; auch ist die Massenzuordnung des Molybdäns von etwa 60 Tagen Halbwertszeit noch unsicher. Man kann annehmen, daß die beiden letztgenannten Molybdän-Isotope zu den Massenzahlen 102 und 104 gehören. Möglicherweise könnte der 60-Tage-Körper ein Isomeres zu einem stabilen Molybdän sein. Ob bei der Spaltung auch ein Molybdän-Isotop der Masse 103 entsteht, ist ungewiß.

Die 14-Min.-Aktivität beim Element 43 gehört als Folgeprodukt des 14-Min.-Molybdäns zur Massenzahl 101, während die mit etwa 15 Min. angegebene Aktivität als Muttersubstanz des 4-Stdn.-Rutheniums zur Masse 105 gehört. Sicherlich sind die Isotope von 14 Min. und etwa 15 Min. Halbwertszeit nicht ein und dasselbe Isotop, da nur der $\sim 15$-Min.-Körper in das 4-Stdn.-Ruthen zerfällt. Die vermutlich ebenfalls bei der Spaltung noch entstehenden Isotope mit den Massenzahlen 102, 103 und 104 sind bis jetzt unbekannt, ihre Halbwertszeiten dürften sehr kurz oder sehr lang sein.

Beim Ruthen steht nur das 4-Stdn.-Ruthen mit der Massenzahl 105 sicher fest. Aus den weiter aufgefundenen Isotopen von 4 Min. und 1 Jahr Halbwertszeit $^{8}$ lassen sich keine aktiven Palladium-Isotope abtrennen. Das berechtigt zu der Annahme, daß die aus ihnen nachgebildeten Rhodium-Aktivitäten in stabile Palladium-Isotope übergehen. Palladium besitzt nun stabile Isotope bei der Masse 106, 108 und 110. Daher ist anzunehmen, daß die oben genannten RuthenIsotope zu diesen Massen gehören. Die Existenz einer weiteren Ruthen-Aktivität von $\sim 40$ Tagen ist sicher; ihre Massenzahl ist allerdings noch nicht angebbar.

Für die bisher bei der Spaltung aufgefundenen Rhodium-Isotope von 34 Stdn., 25 Min. und 40 Sek. ${ }^{8}$

8 W. Seelmann-Eggebert, Naturwiss. [1946] (im Druck). ist der genetische Zusammenhang geklärt. Für ihre Massenzuordnung gilt dasselbe wie das im vorigen Absatz über ihre Muttersubstanzen Gesagte. Weitere aktive Rhodium-Isotope mit den möglichen Massenzahlen 107, 109 und 110 und weiteren Massenzahlen sind noch unbekannt. Vielleicht besitzen auch diese kurze Halbwertszeiten, denn bisher wurde nach kurzlebigen Körpern nicht gesucht, da die schnelle chemische Abscheidung des Rhodiums aus Uran schwierig ist.

Die beiden in Tab. 1 aufgeführten Palladium-Isotope von 26 Min. und 17 Stdn. konnten den Massenzahlen 111 und 112 zugeordnet werden. Der bekannte 13-Stdn.-Körper mit der Massenzahl 107 oder 109 ist bisher bei der Spaltung nicht gefunden worden. Sein Erkennen ist unter den Spaltprodukten des Urans, durch die gleichfalls entstehende Reihe 17-Stdn.Palladium $\rightarrow$ 3,2-Stdn.-Silber $\rightarrow$ Cadmium stabil, erschwert. Weitere evtl. noch vorhandene PalladiumIsotope der Massen 107 oder 109, 113 und höherer Massen sind unter den Spaltprodukten noch nicht festgestellt worden und auch von anderen Kernprozessen her nicht bekannt. Das noch hypothetische zweite aktive Palladium-Isotop der Masse 107 oder 109 ist wohl als äußerst langlebig anzusehen, da es noch nicht gelungen ist, es beim Bestrahlen von Palladium mit verlangsamten Neutronen nachzuweisen.

Beim Silber, dem Element 47, sind drei Isotope mit 7,5 Tagen, 3,2 Stdn. und 20 Min. Halbwertszeit festgestellt worden; die Massenzahlen der beiden ersten sind durch ihren genetischen Zusammenhang als Tochtersubstanzen bekannter Palladium-Isotope gesichert. Die Massenzuordnung des 20-Min.-Silbers ${ }^{8}$ ist nicht frei von Willkür. Ein weiteres Silber-Isotop von etwa 3 Min. ${ }^{8}$ Halbwertszeit ist noch nicht näher untersucht worden. Es besitzt sicher keine langlebige Muttersubstanz; ob es in ein stabiles oder aktives Cadmium-Isotop übergeht, ist gleichfalls noch nicht zu entscheiden. Beide Körper wurden vorläufig bei den Massen 114 und 116 aufgeführt. Die Existenz weiterer Silber-Aktivitäten ist wahrscheinlich. (Die bekannten Isomere von 40 und 31 Sek. Halbwertszeit der Masse 107 bzw. 109 sollten ebenfalls unter den Spaltprodukten zu finden sein.)

Beim Cadmium sind gleichfalls drei Isotope bekannt, von denen zwei durch andere Kernprozesse eindeutig festgelegt wurden. Das auch bei der Spaltung aufgefundene 50-Min.-Cadmium gehört als Isomeres zu einem stabilen Cadmium wahrscheinlich zur Masse 111 oder $113^{9}$. Weitere aktive Cadmium-Isotope sind bei der Spaltung noch zu erwarten: Es wurde aber noch nicht nach ihnen gesucht.

Beim Indium ist außer dem bekannten 4,5-Stdn.Isomer des stabilen Indiums 115 noch ein weiteres Isotop von 117 Min. bekannt, welches der Masse 117 zuzuordnen ist. Nach Aussage der Tab. 1 können fünf, evtl. noch mehr aktive Isotope aufgefunden werden. Hierzu ist zu bemerken, daß die analytische Abscheidung des Indiums aus dem Uran zeitraubend ist; hinzu kommt noch, daß die Intensitäten der Uran-

9 J. Matt a u ch, Z. Physik 117, 246 [1941]. 


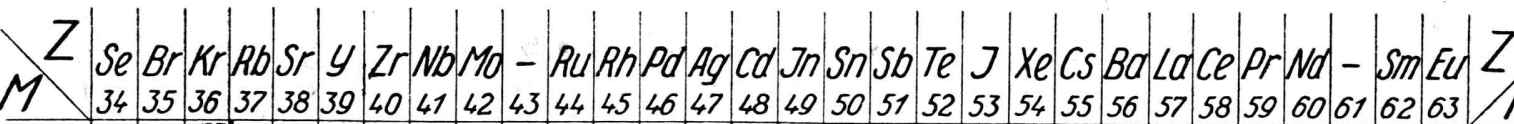

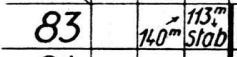

\begin{tabular}{ll|l}
\hline 84 & $30 \%$ & stab \\
\hline
\end{tabular}

$85 \cdot 3 ? 46^{\circ}$ stat

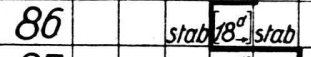

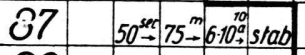

88 175." $18^{\circ}$. scod

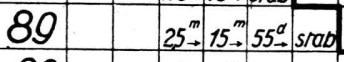

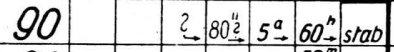

$91 \quad \stackrel{2}{2}-?^{2} \rightarrow 10^{\circ}-57^{50} 5$ stab

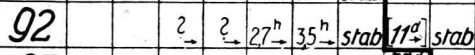

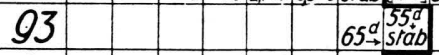

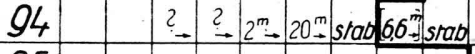

95

96

97

98

99

100

101

102

103

$17_{\rightarrow \rightarrow 5}^{n} \rightarrow$ srad

stat

$s / a b\left[+15^{\bar{m}}\right] s+a b$

119

$\overrightarrow{11}^{\mathrm{d}}$ srab

120

stab $63^{\text {A. }}$ stab

121

$80^{n} \rightarrow \operatorname{srab} s t a b$

122

srab $\left.60^{d}\right]$ stab $\left[-4^{0}\right]$ srab

123

$\mathrm{PO}_{\rightarrow}^{\mathrm{m}} \rightarrow$ ? $\rightarrow$ srab

124

$20^{m} \rightarrow ? \operatorname{srab}\left(13^{a} \rightarrow s / a b\right.$

125

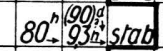

126

127

$\operatorname{stab} 25^{2 \pi} \rightarrow \operatorname{stab}$

128

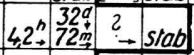

129

$\operatorname{stab} 12,5 * \operatorname{stab}$

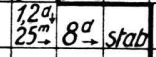

130

$5^{m} \rightarrow 77^{n} \rightarrow 2,4 \div$ stab stab

131

$10^{m} 60^{m} \rightarrow 20^{n} \rightarrow 5^{d} \rightarrow$ stab

132

\begin{tabular}{lll|l|}
$26_{\rightarrow}^{d}$ & 2 & $5 / a b$ \\
\hline
\end{tabular}

$43_{\rightarrow}^{m} 54^{m} \rightarrow \operatorname{srab}\left[3_{\rightarrow}^{h}\right]$ stab

133

$6-10^{a} \rightarrow 100^{2}+?$ ? $\rightarrow$ irab $s$ stab

$67_{\rightarrow}^{n}$ langls stab

$6,6^{m}+10^{m}, 5_{\rightarrow}^{m}$ langl stab

134

$\underset{\rightarrow \text { angi }}{\rightarrow}$ stab $\quad$ stab

$1,8 \rightarrow$ stab stab

135

$14,6^{m} \rightarrow 14^{m} \rightarrow \operatorname{srab}$

$12_{\rightarrow}^{\mathrm{m}} \underset{\mathrm{Kurzi}}{\rightarrow} \operatorname{srab} 210_{\rightarrow}^{d} \mathrm{srab}$

$2^{m} \rightarrow 30_{\rightarrow}^{\mathrm{sec}} 3,8^{\mathrm{m}}$ langl srab

136

104

105

$\left(20^{h}\right)\left(\begin{array}{l}50 \%) \\ 5 r a b\end{array}\right.$

$17_{\rightarrow}^{m} 33_{\rightarrow}^{m} \operatorname{stab}$

137

106

107

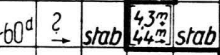

$45^{m}+77^{m} .87^{m}$. sab

138

108

$\sim 5_{\rightarrow}^{m} \sim 15_{\rightarrow}^{m} 4_{\rightarrow}^{h} 34_{\rightarrow \rightarrow}^{h} s t a b$

$\stackrel{?}{\rightarrow} 40^{\sec } 13^{a} \rightarrow 40_{\rightarrow}^{h} \operatorname{srab}$

139

109

110

111

112

113

$\underset{\rightarrow}{a} 40_{\rightarrow}^{\text {sec }} \operatorname{stab}\left[-25^{m}\right]$ stab

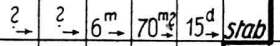

140

114

115

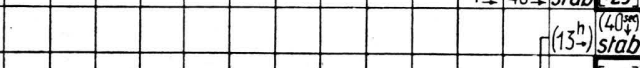

$\stackrel{2}{\rightarrow} \stackrel{?}{\rightarrow} 18_{\rightarrow}^{m} \rightarrow 3,5^{n} \rightarrow$ stab $187^{n}$ stab

141

$4_{\rightarrow}^{m} \rightarrow 25^{m}$ srab $24^{m} \rightarrow$ srab

$15^{m} ? 40^{\mathrm{h}} \rightarrow 13^{\mathrm{d}} \rightarrow \mathrm{srab}$

142

116

117

118

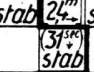

310. $17^{\mathrm{m}} \rightarrow$ stab stab 144

stab $24^{\text {sea }}$ stab

$-L_{4 \rightarrow}^{n ?} \rightarrow 100^{d}+5 r a b$

$-15_{\rightarrow}^{\mathrm{m}}-25^{\mathrm{m}} \rightarrow$ stab

145

$26_{\rightarrow}^{m} 75_{\rightarrow}^{d} \mathrm{stab}$

146

$17^{n} 32^{n} \rightarrow 5 r a b+64^{n} \mathrm{stab}$

$50^{m}-104 \mathrm{~m}^{\mathrm{m}}$

$20 \mathrm{~m}$ stab $72^{\mathrm{sec}}$

$56^{\text {n } 272^{a} \text { sta }}$

\begin{tabular}{ll|l|l}
\hline $5 .+5 r a b$ & & & 146 \\
& & stab & 147 \\
\hline
\end{tabular}

$\rightarrow 3 \rightarrow$ stab $56 \div$ stab

$170^{m} \mid 117^{m} \mathrm{srab}$

148

sab 149

stat stab 150

stab 151

stab 152

stab 153

stab

stab 154

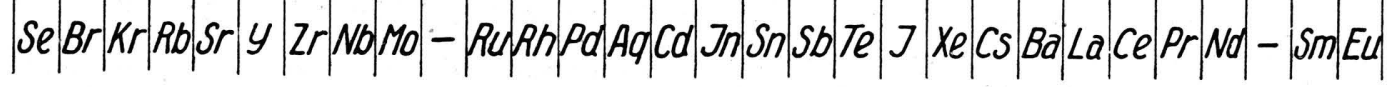

Tab. 1. Die bisher aufgefundenen Uranspaltprodukte. Die starke Linie bedeutet: Grenze, bis zu welcher Spaltprodukte sicherlich gefunden werden können. [], (): Aus anderen Kernprozessen bekannte aktive Isotope, die nach der oben aufgestellten Regel unter den Spaltprodukten nicht [] zu erwarten sind, oder aber () voraussichtlich noch aufgefunden werden. ? Bei der Uranspaltung vorhandenes Isotop, dessen genaue Daten noch nicht bekannt sind. Dünner Druck: Massenzahl steht noch nicht fest. 
spaltprodukte gerade bei den Elementen von Rhodium bis Indium gering sind. Sie treten in der Hauptsache bei Spaltung des Urankerns 238 mit schnellen Neutronen auf. Bisher scheint die Existenz wenigstens eines kurzlebigen Indiums ziemlich sicher ${ }^{7}$. Die Erforschung der aktiven Indium-Isotope ist aber noch keineswegs abgeschlossen.

Die in der Tab. 1 aufgeführten Zinn-Isotope von 11 Tagen, 80 Stdn., 70 Min. und 20 Min. Halbwertszeit sind bei den Massenzahlen 121, 123, 125 und 126 aufgeführt. Ein Grund gerade für diese Einordnung kann nicht gegeben werden. Es existiert darüber hinaus noch ein 4-5-Stdn.-Körper, der entweder ein ZinnIsotop ist oder aber ein aus einem Zinn-Isotop nachgebildetes Antimon-Isotop.

Beim Antimon sind bisher die drei Isotope von 80 Stdn., 4,2 Stdn. und 10 Min. eindeutig bestimmt, während ein 5-Min.-Antimon auf Grund seiner Tochtersubstanzen einer der Massenzahlen 132, 134 oder 136 zugeordnet werden konnte. Es ist wahrscheinlich, daß noch Antimon-Isotope der Massen 125, 126, 128 und 130 entstehen. Genauere Aussagen sind noch nicht zu machen. Es sei noch darauf hingewiesen, daß die schnelle chemische Trennung des Antimons vom Zinn, wenn nur schwache Aktivitäten zur Verfügung stehen, nicht einfach ist.

Alle in Tab. 1 aufgenommenen Tellur-Isotope liegen sicher fest, bis auf das 2-Min.-Tellur, bei welchem noch nicht bekannt ist, in welches Jod-Isotop es zerfällt. Auch ist es nicht sicher, ob das durch $n$, $\gamma$-Prozeß bekannte 90-Tage-Isomer bei der Masse 127 sich unter den Uranspaltprodukten befindet; seine Auffindung ist aber wahrscheinlich.

Die zahlreich auftretenden $J o d$-Isotope bieten in bezug auf ihre Einordnung in das System der Atomkerne keine Schwierigkeiten mehr. Es fehlt bis heute nur das Jod-Isotop der Masse 129, welches als Tochtersubstanz des 30-Tage- bzw. 72-Min.-Tellurs sicher noch aufgefunden wird. Die Halbwertszeit dieses Körpers dürfte extrem lang sein.

Auch für die zahlreich nachgewiesenen XenonIsotope konnten die Massenzahlen festgestellt werden. Nur bei drei Isotopen mit den Massenzahlen 140, 141 und 142 konnten wegen ihrer sehr kurzen Lebensdauer keine genaueren Halbwertszeiten gemessen werden, obwohl ihre Existenz sicher ist. Die Zuordnung zu den Massen 141 nd 142 ist willkürlich.

Bei den Cäsium-Isotopen erfolgt die Zuordnung zu den einzelnen Massenzahlen •in ähnlicher Weise. Die Cäsium-Isotope der Massen 135 und 137 sind sehr langlebig; wenigstens eines von ihnen ist bekannt. Die genaue Halbwertszeit ist noch nicht angebbar. Sie ist jedenfalls größer als 5 Jahre ${ }^{7}$. Die Maximalenergie ihrer Strahlung ist gering. Für die CäsiumIsotope, welche aus den entsprechenden Xenon-Isotopen der Massen 140, 141 und 142 entstehen müssen, gilt dasselbe wie für die Xenon-Isotope der gleichen Masse. Ihre Existenz ist auf Grund des genetischen Zusammenhanges sicher begründet, wenn auch ihre Lebensdauer, welche äußerst kurz ist, nicht bestimmt werden kann.
Die Zuordnung der Barium-Isotope von 87 Min. und 13 Tagen ist gut begründet, während die Zuordnung des 6-Min.- und des 18-Min.-Körpers zu den Massen 141 und 142 nur vermutet werden kann. Für das Vorliegen weiterer Barium-Isotope ist bisher keine Andeutung vorhanden, obgleich wegen der Existenz vieler wohl sicher primärer Krypton-Isotope eine gleiche Anzahl primärer Barium-Isotope auftreten sollte, da die Summe der Kernladungen von Krypton und Barium 92, also die des Urans, ergibt.

Die aus den Barium-Isotopen der Massenzahlen 140, 141 und 142 entstehenden isobaren Lanthan-Isotope von 40 Stdn., 70 Min. und 3,5 Stdn. sind durch ihren genetischen Zusammenhang festgelegt, ohne daß die Zuordnung zu den Massenzahlen 141 und 142 gesichert ist. Ein weiteres Lanthan-Isotop mit einer Halbwertszeit von etwa 15 Min. wurde der Masse 143 zugeschrieben. Seine Entstehung aus einem Xenon ist nicht nachgewiesen worden. Dieser Befund ist im Hinblick auf die Zusammenstellung der Bruchstückpaare interessant, denn das Lanthan ist der Ordnungszahl nach das zu einem Brom gehörende zweite Bruchstück. $\mathrm{Zu}$ den vier, unter Umständen sogar fünf auftretenden Brom-Isotopen sollte die gleiche Anzahl primärer Lanthan-Isotope gehören. Sie sollten wohl alle eine kurze Lebensdauer besitzen. Verschiedene kurzlebige Isotope von „Seltenen Erden“ konnten nachgewiesen werden ${ }^{10}$, ohne daß es gelang, Massenzahl oder Ordnungszahl zu idęntifizieren.

Beim $C e r$ sollten demnach nur dann primäre aktive Isotope zu erwarten sein, wenn Selen als primäres Spaltelement nachgewiesen werden kann. Bisher sind fünf aktive Cer-Isotope bekannt. Ihre Massenzuordnung ist bis auf das Cer von 40 Stdn. Halbwertszeit bei der Masse 143 willkürlich. Die übrigen von 15 Tagen, 310 Tagen, 4 Stdn. und 15 Min. sind vorläufig den Massen 141, 144, 145 und 146 zugeordnet worden. Nach den Bemerkungen ïber die Lanthan-Isotope ist evtl. noch mit einem weiteren Cer-Isotop wohl bei der Masse $147 \mathrm{zu}$ rechnen, außer den noch zu erwartenden primären Cer-Isotopen schwacher Intensitäten (s. u.).

Nach Aussage der Tab. 1 sollten Cer-Isotope mit den Massenzahlen 143 bis 147 in aktive Isotope des Praseodyms übergehen. Es sind schon vier aktive Isotope von 13 Tagen, 17 Min., 100 Tagen und 25 Min. bekannt. Für das 13-Tage-Praseodym wurde der genetische Zusammenhang mit dem 40-Stdn.-Cer wahrscheinlich gemacht ${ }^{11}$. Hingegen ist die Zuordnung des „Praseodyms“ von 100 Tagen als Tochtersubstanz des 4-Stdn.-Cers einstweilen willkürlich. Bei den beiden anderen ist der genetische Zusammenhang zu den Muttersubstanzen eindeutig.

Nimmt man die Existenz eines fünften aktiven Brom-Isotops der Masse 86 bei der Uranspaltung an, so muß nicht nur, wie beim Cer erwähnt, noch je ein aktives Lanthan- und Cer-Isotop auftreten, sondern als aktive Tochtersubstanzen auch Isotope des Prase-

$10 \mathrm{H}$. G öt t e , Z. Naturforschg. 1, 377 [1946].

11 M. L. P o ol u. K u r batov, Physic. Rev. 63, 463 [1943]. 
odyms, Neodyms und des Elements 61. Ein experimenteller Hinweis hat sich noch nicht ergeben. Die Klärung dieser Frage erfordert zweifellos außer starken Neutronenquellen auch rasche analytische Trennmöglichkeiten der verschiedenen auftretenden Seltenen Erden, welche noch weiter entwickelt werden müßten.

Betrachtet man abschließend alle Uranspaltisotope, die einer bestimmten Masse zugeordnet werden konnten, so zeigt sich eine einigermaßen gute Übereinstimmung mit den Ergebnissen der mit physikalischen Methoden durchgeführten Untersuchungen. Nur an der unteren und vor allem an der oberen Grenze der auftretenden Massenzahlen bestehen noch Diskrepanzen. Auf Grund vieler chemischer Untersuchungen (allerdings standen nur sehr schwache Neutronenintensitäten zur Verfügung) ist das Bruchstück mit der kleinsten Masse das ${ }^{83}$ Brom. Dieses Brom entsteht aus einem 30-Min.-Selen, welches bei Bestrahlung von Selen mit langsamen Neutronen gebildet wird. Trotz Suchens ist dieses 30-Min.Selen unter den Spaltprodukten nicht aufgefunden worden. Natürlich ist das bisherige Nichtauffinden dieses Selen-Isotops und anderer Isotope kleinerer Masse kein Beweis dafür, daß nicht noch Isotope mit kleinerer Massenzahl als 83 bei der Uranspaltung auftreten können, denn diese könnten sofort als stabile Isotope entstehen, oder aber mit so geringer Intensität gebildet werden, daß ihr chemischer Nachweis noch nicht glücken konnte. Es handelt sich hierbei um Isotope von Elementen kleinerer Ladungszahl als 35 .

Das gleiche gilt ähnlich auch für die obere Massengrenze der Spaltprodukte, bei der das schwerste chemisch nachgewiesene Spaltprodukt die ungefähre Massenzahl 146 beim Praseodym besitzt. Der chemische Nachweis bei den Seltenen Erden ist schwierig und deshalb noch nicht so genau untersucht wie beim Brom. Es muß aber die Existenz von mindestens 6 bis 7 weiteren Massenzahlen über 146 angenommen werden. Denn zu den bekannten 4 Brom- und 9 Kryptonisotopen gehören als schwere Bruchstücke Atomkerne mit einer größeren Ordnungszahl als der des Cäsiums mit Massenzahlen bis zu 151 oder sogar bis zur Massenzahl 156, je nachdem welchem Spaltprozeß man das Entstehen der genannten Spaltisotope zuschreibt und wieviele Neutronen bei der Spaltung direkt frei werden. Die physikalischen Messungen haben darüber hinaus Massenzahlen bis zu 170 ergeben. Diesen Massen- zahlen entsprechen aktive Isotope von Elementen mit Ordnungszahlen größer als der des Praseodyms.

Versucht man auf Grund der Tab. 1 der Spaltisotope festzustellen, welche Bruchstücke als Paare zusammengehören, so stößt dieser Versuch auf große Schwierigkeiten. Will man die Spaltung des Kerns damit erklären, daß zwei Spaltkerne gebildet werden, deren Ordnungszahlen zusammen 92 ergeben, so muß man bei fast allen Elementen eine Reihe von noch nicht nachgewiesenen Isotopen annehmen. Diese Hypothese scheint durch das Vorhandensein so vieler kurzlebiger Edelgas-Isotope gestützt, denn nur bei gasförmigen Elementen kann es gelingen, Isotope abzutrennen und eindeutig als solche zu identifizieren, wenn die Lebensdauer nur wenige Sekunden oder gar nur Bruchteile von Sekunden beträgt. Eine andere Stütze ist das immer neue Auffinden weiterer kurzlebiger Isotope, wenn die Zeitdauer der chemischen Abtrennung verkürzt werden konnte.

Eine weitere Erklärung wäre die Möglichkeit, daß stabile Isotope als Spaltstücke auftreten, was jedoch eine einseitige Verteilung des Neutronenüberschusses bedeuten würde. Die Annahme einer ungleichen Neutronenverteilung wiederum erscheint wenig wahrscheinlich.

Auch die Abspaltung von Protonen oder von Heliumkernen könnte die vorhandenen Tatsachen erklären, aber weder Protonen noch Spaltungs$\alpha$-Teilchen sind nachgewiesen worden ${ }^{12}$.

Ein weiterer Versuch, das Fehlen einer genügend großen Zahl korrespondierender Spaltstücke zu erklären, wäre die Annahme, daß die primären Spaltstücke nicht nur 'am Anfang einer isobaren Reihe auftreten können, sondern auch sofort mit einer zwar gleichen Masse, aber höheren Ordnungszahl, als es dem ersten Glied einer isobaren Reihe entspräche. Nach einem solchen Effekt wurde bereits gesucht. Beim 40-Stdn.-Lanthan wurde festgestellt, daß die gesamte Aktivität an 40-Stdn.-Lanthan nur über den Zerfall des 13Tage-Bariums entsteht ${ }^{7}$. Beim 9,4-Stdn.-Xenon

12 Im November 1946 ist inzwischen die Mehrfachspaltung des Urans, bei welcher auch ein sehr leichter Kern gebildet wird, was schon K. L a r k-Horowitz u. $\mathrm{S}$ c h r e i b e r, Physic. Rev. 60, 156 [1941], vermuteten, von $\mathrm{T}$ sien San Tsiang, Raymond Chastel, Ho Zah-Wei u. Léopold Vigneron, C. R. hebd. Séances Acad. Sci. 223, 986 [1946], und H o Z a hWei, Tsien San Tsiang, Lé opold Vigneron u. R a y mond Chastel, ebenda 223, 1119 [1946], bestätigt worden. 
sieht es hingegen nicht so àus, als ob die gesamte 9,4-Stdn.-Xenon-Aktivität über das 6,6-Stdn.-Jod gebildet würde ${ }^{7}$; allerdings ist der Effekt noch nicht ganz sicher, auf jeden Fall aber äußerst klein. Die Schwierigkeit, einen solchen Effekt nachzuweisen, liegt darin, daß starke Quellen nötig sind, weil die Halbwertszeiten in den allermeisten Fällen für die bèabsichtigte Untersuchung äußerst ungünstig sind, so daß nur sehr kurz bestrahlt werden kann.

Die bisher durchgeführten Untersuchungen über die Intensitätsunterschiede bei den einzelnen isobaren Reihen bestätigen im wesentlichen die Ergebnisse der physikalischen Messungen. Einzelne isobare Reihen scheinen gegenüber ihren Nachbarn bevorzugt gebildet zu werden. Vielleicht ist auch die Energie der die Spaltung auslösenden Neutronen von ausschlaggebender Bedeutung für die Intensitätsverhältnisse der Spaltprodukte. Diese feineren Intensitätsunterschiede nahe benachbarter Reihen vermuteten schon A. Fla mmersfeld, W. Gentner und A. Jensen ${ }^{3}$. Genauere Angaben können noch nicht gemacht werden, da die Verschiedenheit der Bruchstücke bei der Spaltung der beiden Uranisotope mit langsamen und energiereichen Neutronen die Untersuchung erschwert.

Abschließend möchten wir betonen, daß diese Arbeit nur einen sehr vorläufigen Überblick über die Chemie der Uranspaltung geben soll und daß noch eine große Zahl von Untersuchungen nötig ist, um die vielen Fragen endgültig zu klären.

\title{
K-Einfang beim natürlich aktiven Cassiopeium
}

\author{
Von ARnold Flammersfeld \\ Aus dem Kaiser-Wilhelm-Institut für Chemie, Tailfingen \\ (Z. Naturforschg. 2a, 86-89 [1947]; eingegangen am 11. Dezember 1946)
}

\begin{abstract}
Die von dem natürlich aktiven ${ }^{176} \mathrm{Cp}$ ausgesandte durchdringende Strahlung wurde durch Absorption in Kupfer, Cadmium und Blei untersucht. Außer der bereits bekannten $\gamma$-Strahlung von $260 \mathrm{KeV}$ wurde noch eine $K$-Strahlung gleicher Intensität nachgewiesen. Durch Vergleich mit den Ergebnissen von W. B othe am 6,8-Tage-Cp wurden beide Strahlungen auf $K$-Einfang zurückgeführt, so daß auch der Grundzustand ${ }^{176} \mathrm{Cp}$ dual zerfällt. Der $K$-Einfang ist $2 \pm 0,4$-mal so häufig wie der $\beta$-Übergang, so daß die Halbwertszeit des Isotops ${ }^{176} \mathrm{Cp}$ nur ${ }^{1 / 3}$ des von L i b b y aus der $\beta$-Aktivität bestimmten Wertes, nämlich $2,4 \cdot 10^{10}$ Jahre, beträgt.
\end{abstract}

\section{Problemstellung}

$\mathrm{D}$ as Element Cassiopeium besteht aus zwei Isotopen der Massenzahl 175 (Häufigkeit $97,5 \%)$ und $176(2,5 \%)^{1}$. Auf Grund der Isobarenregeln konnte $\mathrm{M}$ a t t a $\mathrm{u} \mathrm{ch}$ für das letztere Instabilität vorhersagen ${ }^{2}$, worauf $\mathrm{He}$ y d e $\mathrm{n}$ und Wefelmeie ${ }^{3}$ dessen Aktivität nachwiesen. Das Isotop 176 zeichnet sich durch einen bemerkenswert hohen Spin $i \geq 7$ aus $^{4}$, und gelegentlich der Aufstellung der Kernisomerenregeln folgerte $\mathrm{M} \mathrm{at}$ t a u $\mathrm{ch}$, daß mindestens ein Isomer $\mathrm{zu}$ diesem Kern möglich sein müßte ${ }^{5}$. Eine daraufhin

\footnotetext{
1 J. Mattauch u. H. Lichtbla u, Z. Physik 111, 514 [1939].

2 J. Matt a u ch, Naturwiss. 25, 812 [1937].

3 M. He y d en u. W. W e f e l m e i e r, Naturwiss. 26, 612 [1938].
}

unternommene Untersuchung der durch Neutronen verursachten künstlichen Aktivitäten des Cassiopeiums hatte ergeben, daß die eine Halbwertszeit von 6,8 Tagen vom Isomer zum natürlich aktiven ${ }^{176} \mathrm{Cp}$ herrührt ${ }^{6}$.

Anläßlich dieser Untersuchung konnten F lammersfeld und Mattauch auch eine mit der natürlichen Aktivität verbundene $\gamma$-Strahlung der Energie $0,260 \mathrm{MeV}$ auffinden, als deren Ursprung entweder $K$-Einfang (also dualer Zerfall des ${ }^{176} \mathrm{Cp}$ ) oder komplexer $\beta$-Zerfall vermutet wurde.

Neuerdings wurden die Strahlungen künstlich erzeugter Cp-Isotope von B othe mit stärkeren

${ }^{4}$ H. S chü l e r u. H. G o l l now, Z. Physik 113, $1[1939]$.

5 J. M a t t a u ch, Z. Physik 117, 246 [1941].

6 A. F l a m m e r s f eld u. J. M at t a u ch, Naturwiss. 31, 66 [1943]; Halbwertszeit nach W. Both e ${ }^{7}$. 